\title{
DIFFERENT TYPES OF MAPS IN DYNAMICAL SYSTEMS ROUTES TO CHAOS IN MATHEMATICS
}

\section{HENA RANI BISWAS ${ }^{1}$, MD. AL-AMIN ${ }^{1}$, MD. MONIRUL ISLAM SUMON $^{2}$ and FOUZIA SHARMIN ${ }^{3}$}

${ }^{1}$ Department of Mathematics

University of Barishal

Barishal-8200

Bangladesh

e-mail: biswas.hena@yahoo.com

2Department of Business Administration

Z. H. Sikder University of Science and Technology

Shariatpur

Bangladesh

${ }^{3}$ Department of Basic Science

World University

Dhaka-1230

Bangladesh

\begin{abstract}
In this paper, we have discussed the general concepts of chaos theory in dynamical systems and main characteristics of chaos. We have described manipulating chaos, different definitions of chaos and their interrelationship in this study. In this work we have found that one dimensional maps with complicated dynamical behaviour. We have solved some problems of tent map
\end{abstract}

2010 Mathematics Subject Classification: 35B32.

Keywords and phrases: chaos theory, Lyapunov exponent, topological transitive.

Received April 26, 2020; Revised June 2, 2020

(C) 2020 Scientific Advances Publishers 
and after solving those problems we observed that tent map is chaotic. We also discussed the bifurcation of one dimensional map and sketch bifurcation diagrams by using MATHEMATICA.

\section{Introduction}

Chaos is one of the few concepts in mathematics which cannot usually be defined in a word or statement. Most dynamical systems are considered chaotic depending on the either the topological or metric properties of the system. Chaos theory is a branch of mathematics focusing on the behaviour of dynamical systems that are highly sensitive to initial conditions. Chaos theory is the study of how systems that follow simple, straightforward, deterministic laws can exhibit very complicated and seemingly random long term behaviour. In common usage, "chaos" means "a state of disorder". However, in chaos theory, the term is defined more precisely. Although there has been no universally accepted mathematical definition of chaos, it is generally believed that if for any system the distance between the nearby points increases and the distance between the far away point's decreases with time, the system is said to be chaotic. Chaotic dynamical systems constitute a special class of dynamical systems. Hence a dynamical system is chaotic if the orbit of it (or a subset of it) are confined to a bounded region, but still behave unpredictably.

The first experience with what is now called chaos was with Henry Poincare, the famous French mathematician in the early 1900's. Poincare is considered as the last of the Universalist (people who made major contributions to all major and known areas in mathematics) in mathematics. Edward Lorenz from MIT is known and acknowledged as the modern father of chaos theory. He was a meteorologist and had so much interest in long term predictions of the weather. Edward Lorenz describes chaos in these words "Chaos is when the present determines the future but the approximate present does not approximately determine the future". He further explained that the unpredictability nature of the weather is because we can only measure the weather approximately. He 
only realized from his work graphically that though they have almost same starting points, the difference in their final points given the same number of iterations was so wide and unimaginable. Edward Lorenz after careful consideration and scrutiny realized that the two initial input values differ by decimal points. The output from his machine had three decimal places compare to the six decimals of his original inputs. This small numerical difference has contributed to great difference in his computations. If his computer is not faulty then perhaps, there is something mathematicians are failing to acknowledge; a small change in input produces a very great difference in the end. And chaos has been born and the rest followed. Chaos theory was discovered in 1963 though Lorenz had observed the phenomenon two years earlier. Chaotic dynamics was made popular by the computer experiments of Robert May and Mitchell Feigenbaum on a mapping known as the logistic map. The remarkable feature of the logistic map is in the simplicity of its form (quadratic) and the complexity of its dynamics. The logistic map became a paradigm of chaotic behaviour in the 1970s. In 1964 Lorenz published a paper on the logistic map, essentially noting that even with such a simple equation as the logistic map, non-periodic behaviour existed. He drew an analogy with the prediction of climate (that is the long term statistical properties of the weather) and concluded that such prediction may not be possible.

Chaos is an interdisciplinary theory stating that within the apparent randomness of chaotic complex systems, there are underlying patterns, self-similarity, fractals, and initial point known as sensitive dependence on initial conditions. A hallmark of chaotic systems is sensitive dependence on initial conditions. This means that if two copies of the system differ by only a very small amount, then after a relatively short period of time, the two systems will diverge and appear very different from each other. Small differences in initial conditions, such as those due to rounding errors in numerical computation, yield widely diverging outcomes for such dynamical systems, rendering long-term prediction of 
their behaviour impossible in general. This happens even though these systems are deterministic, meaning that their future behaviour is fully determined by their initial conditions, with no random elements involved. In other words, the deterministic nature of these systems does not make them predictable. This behaviour is known as deterministic chaos, or simply chaos.

In mathematics, chaos theory are presented through a brief analysis of some interesting dynamical systems which exists one-, two- and threedimensional map like logistic map, tent map, Hénon map, and Lorenz map which exhibits chaotic behaviour. Chaos theory has applications in several disciplines, including meteorology, anthropology, sociology, physics, mathematics, environmental science, computer science, engineering, economics, biology, ecology, and philosophy. Chaotic behaviour exists in many natural systems, such as weather and climate. It also occurs spontaneously in some systems with artificial components, such as road traffic. This behaviour can be studied through analysis of a chaotic mathematical model, or through analytical techniques such as recurrence plots and Poincaré maps.

In 1975, $\mathrm{Li}$ and Yorke [6] gave the first mathematical definition of chaos through the introduction of $\delta$-scrambled set and in 1993, Li [12] introduced the notion of $\omega$-chaos through the introduction of $\omega$-scrambled set. Devaney [4] chaos is another popular type of chaos. Another interesting definition of chaos is generic chaos.

Most Mathematicians and Physicist like Newton and Laplace made indirect contribution to the field of chaos theory. Alexander Lyapunov also made some contribution in the early stages. His was in the study of the instability of fluids and turbulence in fluids or gases. He tried to measure the transition from order to chaos. Other academicians who made useful contributions to the course are: G.D. Birkhoff, A. N. Kolmogorov, M. L. Cartwright, J. E. Little and Stephen Smale among others. Stephen Smale is the only mathematician and specifically pure 
mathematician to have studied chaos. Li and Yorke [6] concluded that period three implies chaos. They discussed how a dynamical system with period three orbits gives an assurance that the system is chaotic. Several approaches and conditions are factored before the construction of any definition of chaos. Devaney [3] has one of the most popular and accepted definitions of chaos in which such systems must exhibit sensitive dependence to initial conditions, topological transitivity, and dense periodic orbits. Later it was proven that if a system is transitive with dense periodic orbits then obviously sensitivity dependence to initial condition is guaranteed [9].

In this paper, we will discuss main characteristics of chaos, different definitions of chaos and their relationships. We also discuss different types of one dimensional maps and fixed and periodic points, bifurcation diagram, cobweb plots, strange attractor and finally discuss their chaotic behaviour.

\section{Mathematical Preliminaries}

In this section, we present some definitions and theorems which are essential for finding chaotic dynamical behaviour of one dimensional map in the next section.

Definition 2.1 (Dense). Suppose $X$ is a set and $Y$ is a subset of $X$. Then we say that $Y$ is a dense in $X$ if for any point $x \in X$, there is a point $y$ in the subset $Y$ arbitrary close to $x$ [3].

Definition 2.2 (Transitive). Let $(X, U)$ be a dynamical systems. Then $f$ is a transitive if for any two non-empty open subsets $U$, of $X$ there exists $n \geq 0$ such that $f^{n}(U) \cap V \neq \varnothing[3]$.

Definition 2.3 (Sensitive). Let $(X, f)$ be a dynamical systems where $X$ is a metric $d$. Then $f$ is sensitive if there exists some $\delta>0$ with the property that $\forall x \in X$ and $\forall \varepsilon>0, \exists y \in(x, \varepsilon)$ and $\exists n \in N$ such that $d\left(f^{n}(x), f^{n}(y)\right)>\delta$. These three properties of dense, topological transitivity and sensitivity are the basic ingredients of chaotic system [3]. 
Definition 2.4 (Lyapunov number). Let $f$ be a smooth map of the real line. The Lyapunov number $L(x)$ of the orbit $\left\{x_{1}, x_{2}, x_{3}, x_{4}, \ldots\right\}$ is defined as

$$
L\left(x_{1}\right)=\lim _{n \rightarrow \infty}\left(\left|f^{\prime}\left(x_{1}\right)\right| \ldots\left|f^{\prime}\left(x_{n}\right)\right|\right)^{\frac{1}{n}} \text { if this limit exists. }
$$

The Lyapunov exponent $h\left(x_{1}\right)$ is defined as

$$
\begin{aligned}
h\left(x_{1}\right) & =\lim _{n \rightarrow \infty} \frac{1}{n}\left[\ln \left|f^{\prime}\left(x_{1}\right)\right|+\cdots+\ln \left|f^{\prime}\left(x_{n}\right)\right|\right] \\
& =\lim _{n \rightarrow \infty} \frac{1}{n} \sum_{i=1}^{n} \ln \left|f^{\prime}\left(x_{i}\right)\right| \\
& =\lim _{n \rightarrow \infty} \frac{1}{n}\left[\ln \prod_{i=1}^{n}\left|f^{\prime}\left(x_{i}\right)\right|\right]
\end{aligned}
$$

if this limit exists.

Theorem 2.1. Suppose $F: R \rightarrow R$ is continuous function and has a periodic point of period three. Then $F$ has periodic point of all other periods.

Definition 2.5 (Bifurcation). Let $f_{c}$ be a parameterized family of functions. Then there is a bifurcation at $c_{0}$ if there exists $\epsilon>0$ such that whenever $a$ and $b$ satisfy $c_{0}-\epsilon<a<c_{0}$ and $c_{0}<b<c_{0}+\epsilon$, then the dynamics of $f_{a}$ are different from the dynamics of $f_{b}$. In other words, the dynamics of the function changes when the parameter value crosses through the point $c_{0}$. 


\section{Three Main Characteristics of Chaos}

Chaos theory is a subset of the study of dynamical systems. Chaos theory is concerned with dynamical systems that fulfill three conditions. We will explain each of the conditions for a dynamical system to be considered chaotic.

There is no main mathematical definition for chaos. Instead there are three main characteristic behaviours that are associated with a chaotic system. They are (i) sensitivity to initial condition, (ii) density of unstable periodic orbits in a chaotic attractor, and (iii) topological transitivity.

- Sensitivity to initial conditions: The first requirement for a dynamical system to be considered chaotic is that it must be sensitive to the initial condition of the system. Loosely speaking, this means that arbitrarily close start states will eventually become drastically different in their behaviour. We call the values that an initial condition takes on when iterated in a chaotic function a trajectory. Thus, we can say that given two arbitrarily close start states, they will have drastically different trajectories.

- Topologically mixing: The second criterion that a dynamical system must satisfy to be considered chaotic is that it must be topologically mixing. This means that any given open subset of the domain will eventually, intersect with any given open subset of the range of the function.

- Density of periodic orbits: The final criterion to be met in order for a dynamical system to be considered chaotic is that it must have dense periodic orbits if it has periodic orbits. In mathematics, an orbit is simply a collection of points related by iterations of a given function. A periodic orbit is an orbit that oscillates between a finite set of values. Cycles of various lengths are possible in dynamical and chaotic systems. In order for a dynamical system to have dense periodic orbits, any point in the range of the function must be approached arbitrarily close by periodic [7]. 


\section{Different Definition of Chaos}

In this section, we discuss different definition of chaos.

\section{(a) Devaney's definition of chaos}

Let $X$ be a compact metric space. A continuous map $f: X \rightarrow X$ is said to be chaotic [3] on $X$ if $f$ satisfies the following properties:

(i) Periodic points of $f$ are dense in $X$.

(ii) $f$ is one-sided topologically transitive.

(iii) $f$ has sensitive dependence on initial conditions.

(i) and (ii) of the definition of chaotic map are re-written as follows:

(i) $\operatorname{per}(f)$ is dense in $X$.

(ii) For any pair of non-empty sets $U$ and $V$ in $X$ there exists $k \in N$ such that $f^{k}(U) \cap V \neq \varnothing$. This definition of chaos is one of the widely known and accepted definitions in chaos theory. The foundation and basis of the definition is in the systems dependence on initial conditions. Later it was discovered that, once a system is transitive and has periodic orbit being dense, then sensitivity is assured. This makes the foundation of the definition redundant. The property of transitivity and periodic orbits being dense in the set $X$, are invariant under topological conjugation. It points out the fact that the two are topological properties [3].

\section{(b) Li-Yorke definition of chaos}

A dynamical system $(f, I)$ where $f: I \rightarrow I$ is continuous and $I$ is a bounded interval. Then $f$ is chaotic in $I$ in the Li-Yorke sense if $f$ has a periodic point in $I$ of point 3 . 
In other words a dynamical system $(I, f)$ where $f$ is continuous and $I$ is a bounded interval. Then $f$ is chaotic in $I$ in the Li-Yorke, sense if $f$ has periodic point in $I$ of period 3. Let $f: X \rightarrow X$ be a map on a metric space $X$ with metric $d$. The dynamical system $(X, f)$ is said to be Li-Yorke chaotic if there exists a subset $S \subset X$ such that $S$ is uncountable and satisfies:

(i) $\lim \sup _{n \rightarrow \infty} d\left(f^{n}(x), f^{n}(y)\right)>0$ for all $x, y \in S, x \neq y$,

(ii) $\liminf _{n \rightarrow \infty} d\left(f^{n}(x), f^{n}(y)\right)=0$ for all $x, y \in S, x \neq y$,

(iii) $\lim \sup _{n \rightarrow \infty} d\left(f^{n}(x), f^{n}(p)\right)>0$ for all $x \in S, p \in X, p$ is periodic.

In Li \& Yorke's original definition of chaos there is the additional condition that $f$ has periodic points of any period $\mathbb{N}$ [6].

\section{(c) Wiggins definition of chaos}

Let $f: X \rightarrow X$ be continuos map and $X$ be a metric space. The map is considered to be chaotic if

(i) $f$ is topologically transitive,

(ii) $f$ has sensitivity dependence on initial conditions [7].

\section{(d) Lyapunov definition of chaos}

Let $f: \mathfrak{R} \rightarrow \mathfrak{R}$ be a continuous differentiable map. The map $f$ is said to be chaotic if

(i) $f$ is topological transitive,

(ii) $f$ has positive Lyapunov exponent [7].

\section{(e) Knudsen definition of chaos}

Definition. Let $F: X \rightarrow X$ be a continuous map on a metric space $(x, d)$, then the dynamical system $\langle X, F\rangle$ is chaotic according to Knudsen's definition iff

(i) $F$ has dense orbits,

(ii) $F$ is sensitive to initial conditions [7]. 


\section{Interrelationship of Various Types of Chaos}

Devaney chaos implies Wiggin's chaos, Knudsen chaos and Lyapunov chaos. Sensitivity to initial conditions is a common component in all three chaos mentioned above whiles its equivalent of positive Lyapunov is used in Lyapunov's definition. Now, Devaney chaos combines both topological transitivity and the existence of dense orbits. Since both Wiggins chaos, Lyapunov and Knudsen chaos depends on either of the two topological conditions, then Devaney chaos implies them all. A system that is Devaney chaotic has to be Wiggins chaotic, Lyapunov chaotic and Knudsen chaotic. Positive expansive implies Wiggin's chaos, Knudsen chaos and Lyapunov chaos. Wiggin's definition, Knudsen chaos and Lyapunov's definitions satisfies either the condition of transitivity or dense orbits. In relation to Wiggin's chaos, their distinct conditions are positive expansiveness and sensitive dependence to initial conditions. Expansively implies sensitive dependence hence positive expansive chaos implies Wiggin's chaos [7]. In the case of Knudsen chaos, beyond the existence of dense orbit, the distinct condition is also sensitivity. Similarly, since expansively implies sensitive dependence, expansive chaos implies Knudsen chaos. Also for positive Lyapunov exponents, the distinct condition is expansively and positive Lyapunov exponents. Every expansive map has a positive Lyapunov exponent but the converse is not true. As said earlier, if just two orbits separate apart at a point in the iteration, the Lyapunov will be positive but the map might not necessarily be expansive [11].

Wiggin's chaos and Knudsen chaos imply each another and are quite similar or equivalent. They share a common property of transitivity. They also share an equivalent property of sensitivity and positive Lyapunov exponents. The difference in the two definitions is the space on which it is defined. Wiggins considers a continuous map on a metric whiles Lyapunov deals with differentiable maps Knudsen chaos shares an equivalent property of sensitivity dependence to initial condition and 
positive Lyapunov exponents with Wiggins definition and Lyapunov's definition. In some systems, transitivity is equivalent to dense orbits though not always. In such systems, all three chaos are the same apart from the space on which each is defined. Devaney chaos and Li-Yorke chaos are interrelated via topological entropy. Devaney chaos implies positive topological entropy and the converse is not true. Positive topological entropy implies Li-Yorke chaos and here too the converse does not hold. According to the law of transitivity in analysis Devaney chaos implies Li-Yorke chaos. On the interval map Devaney chaos is the strongest whereas Li-Yorke chaos is the weakest. Maps with discontinuity are usually not chaotic.

From above, Devaney chaos implies Wiggin's chaos, Lyapunov chaos and Knudsen chaos. Positive expansive chaos implies Wiggin's chaos, Lyapunov chaos and Knudsen chaos. Possibly then, there should be a link between the two. The two definitions share two common property of topological transitivity and dense periodic orbit. Positive expansively is a stronger property than sensitive dependence to initial conditions. Since positive expansively implies sensitive dependence, the positive expansive chaos implies Devaney chaos [3].

\section{Different Types of Maps Routes to Chaos}

The quadratic map (Logistic map) is defined by the equation $x_{n+1}=f_{a}\left(x_{n}\right)=a x_{n}\left(1-x_{n}\right)$, where $x_{n}$ is a number between zero and one, and represents the population at year $n$, and hence $x_{0}$ represents the initial population (at year 0 ) and $a$ is a positive number, and represents a combined rate for reproduction and starvation.

We will consider the quadratic map $f_{a}(x)$ on the interval $0<x<1$, as a function of the parameter $a$. With $a$ between 0 and 1, the population will eventually die, independent of the initial population. With $a$ between

1 and 2 , the population will quickly approach the value $1-\frac{1}{a}$, 
independent of the initial population. With $a$ between 2 and 3 , the population will also eventually approach the same value $1-\frac{1}{a}$, but first will fluctuate around that value for some time. The rate of convergence is linear, except for $a=3$, when it is dramatically slow, less than linear. For $a>3$ changes to repelling and a 2 -cycle is born. The system exhibits some interesting phenomena [4], which cannot be observed from the continuous logistic system. For $3<a<1+\sqrt{6} \approx 3.45$, the two cycle is stable the population may oscillate between two values forever. For $a=1+\sqrt{6} \approx 3.544090$ the 2 cycle becomes unstable and a stable 4-cycle is born then the population may oscillate between four values forever. For $1+\sqrt{6}<a<1+2 \sqrt{2}=5.544090$, the 4 -cycle becomes unstable and a stable 8 -cycle is born, when $a$ is slightly bigger than 3.54 then 8 than 16 , 32 etc.

In general, a stable $2^{k}$ cycle is born at $a_{k}$ and becomes unstable at $a_{k+1}$, where

$$
\begin{aligned}
& a_{1}=3, \\
& a_{2}=3.449 \ldots \ldots, \\
& a_{3}=3.54409 \ldots, \\
& a_{4}=3.5644 \ldots \ldots, \\
& a_{5}=3.568759 \ldots, \\
& a_{6}=3.569692 \ldots, \\
& a_{7}=3.5698 \ldots, \\
& a_{8}=3.569946 \ldots .
\end{aligned}
$$


It should be clear that as $n$ goes large these values are approaching a limit

$$
a_{\infty}=\lim _{n \rightarrow \infty} a_{n}=3.569946 \ldots
$$

It means that an $\infty$ cycle for the value of $a$. Note that the successive bifurcations come faster and faster. The convergence is essentially geometric; the limit of large $n$, the distance between successive transitions shrinks by a constant factor $\delta=\lim _{n \rightarrow \infty} \frac{a_{n}-a_{n-1}}{a_{n+1}-a_{n}}=4.6692016091 \ldots$

At $a$ approximately 3.57 is the onset of chaos, at the end of the perioddoubling cascade. Most values beyond 3.57 exhibit chaotic behaviour, but there are still certain isolated ranges of $a$ that show non-chaotic behaviour; these are sometimes called islands of stability [1]. For instance, beginning at 3.83 there is a range of parameters $a$ which show oscillation among three values, and for slightly higher values of $a$ oscillation among 6 values, then 12 etc.

The development of the chaotic behaviour of the logistic sequence as the parameter $a$ varies from approximately 3.5699 to approximately 3.8284 is sometimes called the Pomeau-Manneville scenario, which is characterized by a period phase interrupted by bursts of aperiodic behaviour.

\section{Tent Map}

Define as iterative map by $x_{n+1}=T_{b}\left(x_{n}\right)=\left\{\begin{array}{ll}b x_{n} & \text { for } x_{n}<\frac{1}{2} \\ b\left(1-x_{n}\right) & \text { for } \frac{1}{2} \leq x_{n}\end{array}\right.$, where $b$ is a positive real constant, and $x_{n} \in[0,1]$. Although the form of the tent map is simple and the equations involved are linear, for certain parameter values, this system can display highly complex behaviour and even chaotic phenomena. For certain parameter values, the mapping undergoes stretching and folding transformations and display sensitivity to initial conditions and periodicity. 
By varying the parameter $b$, the following behaviour is observed:

(i) If $b$ is less than 1 the point $x=0$ is an attractive fixed point of the system for all initial values of $x$.

(ii) If $b$ is greater than 1 the system has two fixed points, are at 0 and the other at $\frac{b}{b+1}$. Both fixed points are unstable. For example, when $b$ is 1.5 there is a fixed point at $x=0.6$ (because $1.5(1-0.6)=0.6$ ) but starting at $x=0.61$, we get $0.61 \rightarrow 0.585 \rightarrow 0.6225 \rightarrow 0.56625 \rightarrow 0.650625 \ldots$

(iii) If $b$ is between 1 and the square root of 2 the system maps a set of intervals between $b-\frac{b^{2}}{2}$ and $\frac{b}{2}$ to themselves. This set of intervals is the Julia set of the map that is the smallest invariant sub-set of the real line under the map. If $b$ is greater than the square root of 2 , these interval merge, as the Julia set is the whole interval from $b-\frac{b^{2}}{2}$ to $\frac{b}{2}$.

(iv) If $b$ is between 1 and 2 the interval $\left[b-\frac{b^{2}}{2}, \frac{b}{2}\right]$ contains both periodic and non-periodic points, although all of the orbits are unstable that is nearby points move away from the orbits rather than towards them.

(v) If $b$ equals 2 the system maps the interval $[0,1]$ onto itself. There are new periodic points with every orbit length within this interval, as well as non-periodic points. The periodic points are dense in $[0,1]$, so the map has become chaotic.

(vi) If $b$ is greater than 2 the map's Julia set becomes disconnected, and breaks up into a cantor set within the interval $[0,1]$. The Julia set still contain an infinite number of both non-periodic and periodic points but almost every point within $[0,1]$ will now eventually diverges towards infinity. The canonical cantor set is the Julia set of the tent map for $b=3$. 
Problem 1. Iterate the tent function numerically and graphically for the following $b$ and $x_{0}$ values:

(I) $b=\frac{1}{2}$ : (i) $x_{0}=\frac{1}{4}$, (ii) $x_{0}=\frac{1}{2}$, (iii) $x_{0}=\frac{3}{4}$.

(II) $b=1:$ (i) $x_{0}=\frac{1}{3}$, (ii) $x_{0}=\frac{2}{3}$.

(III) $b=\frac{3}{2}$ : (i) $x_{0}=\frac{3}{5}$, (ii) $x_{0}=\frac{6}{13}$, (iii) $x_{0}=\frac{1}{3}$.

(IV) $b=2:$ (i) $x_{0}=\frac{1}{3}$, (ii) $x_{0}=\frac{1}{5}$, (iii) $x_{0}=\frac{1}{7}$, (iv) $x_{0}=\frac{1}{11}$.

Solution 1. For simplicity, the iterates will be listed as $\left\{x_{0}, x_{1}, x_{2}, \ldots, x_{n}, \ldots\right\}$. The solutions are as follows:

(I) $b=\frac{1}{2}$

(i) $\left\{\frac{1}{4}, \frac{1}{8}, \frac{1}{16}, \ldots, \frac{1}{4 \times 2^{n}}\right\}$;

(ii) $\left\{\frac{1}{2}, \frac{1}{4}, \frac{1}{8}, \ldots, \frac{1}{2^{n+1}}\right\}$;

(iii) $\left\{\frac{3}{4}, \frac{3}{8}, \frac{3}{16}, \ldots, \frac{3}{4 \times 2^{n}}\right\}$. In each case $x_{n} \rightarrow 0$ as $n \rightarrow \infty$. 


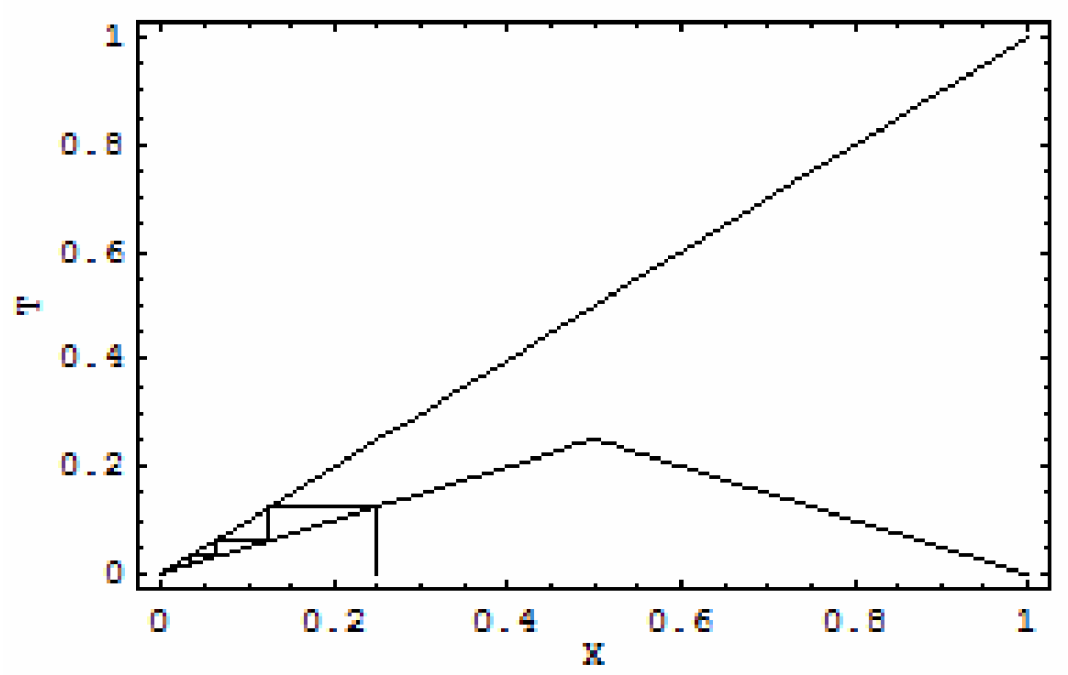

(a)

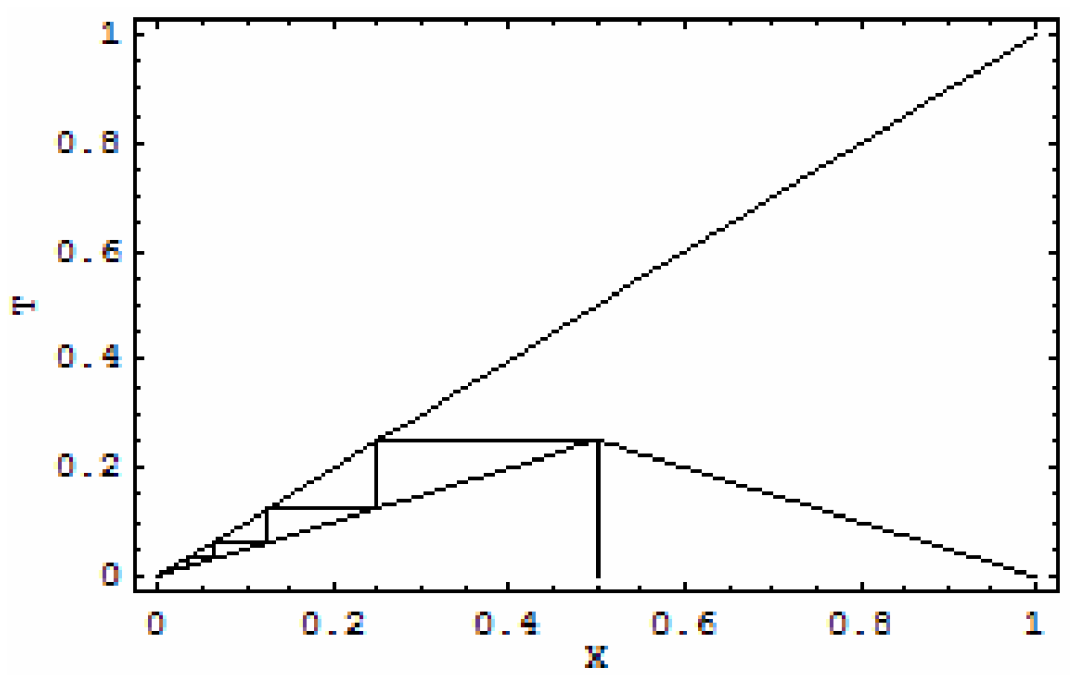

(b) 


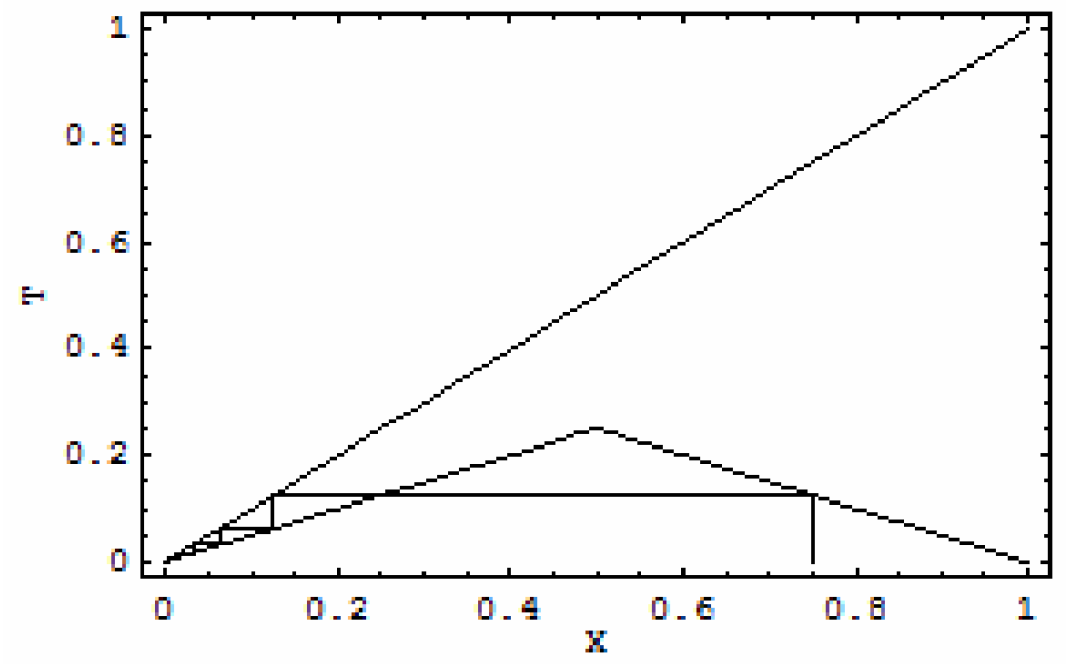

(c)

Figure 1. Graphical iterations when $b=\frac{1}{2}$ : (a) $x_{0}=\frac{1}{4}$, (b) $x_{0}=\frac{1}{2}$, and (c) $x_{0}=\frac{3}{4}$.

(II) $b=1$ :

(i) $\left\{\frac{1}{3}, \frac{1}{3}, \frac{1}{3}, \ldots, \frac{1}{3}, \ldots\right\}$;

(ii) $\left\{\frac{2}{3}, \frac{1}{3}, \frac{1}{3}, \ldots, \frac{1}{3}, \ldots\right\}$.

The orbits tend to points of period one in the range $\left[0, \frac{1}{2}\right]$. 


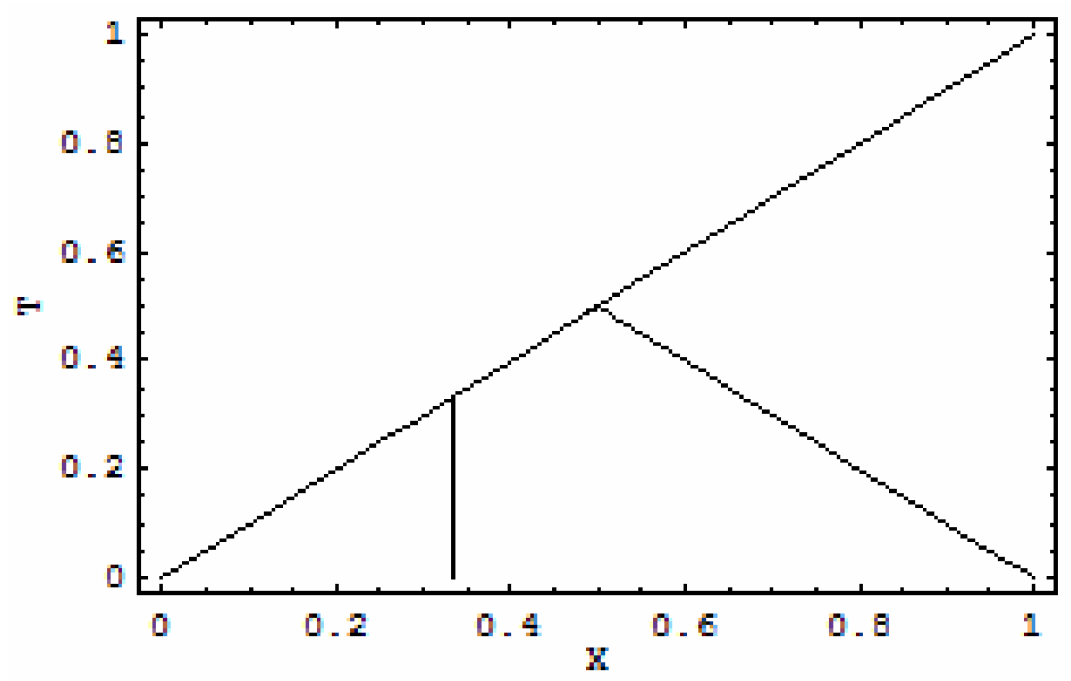

(a)

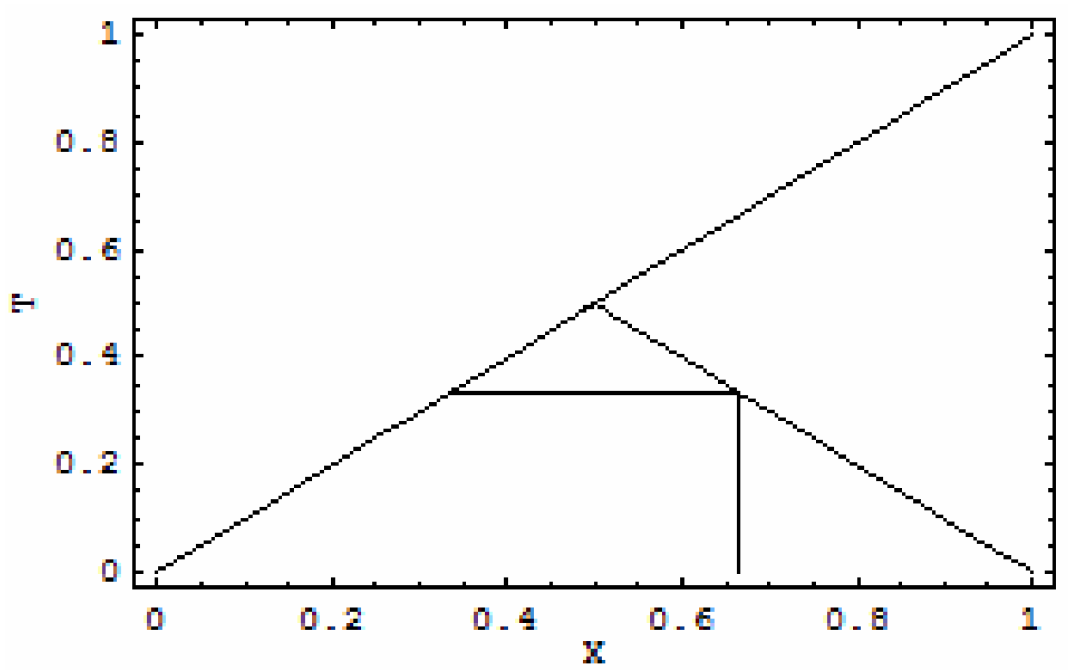

(b)

Figure 2. Graphical iteration when $b=1$ : (a) $x_{0}=\frac{1}{3}$ and (b) $x_{0}=\frac{2}{3}$. 
(III) $b=\frac{3}{2}$ :

(i) $\left\{\frac{3}{5}, \frac{3}{5}, \frac{3}{5}, \ldots, \frac{3}{5}, \ldots\right\}$;

(ii) $\left\{\frac{6}{13}, \frac{9}{13}, \frac{6}{12}, \frac{9}{13}, \ldots, \frac{6}{12}, \frac{9}{13} \ldots\right\}$;

(iii) $\left\{\frac{1}{3}, \frac{1}{2}, \frac{3}{4}, \frac{8}{3}, \frac{9}{16}, \frac{21}{32}, \frac{33}{64}, \frac{93}{128}, \frac{105}{256}, \frac{315}{512}, \frac{591}{1024}, \ldots\right\}$.

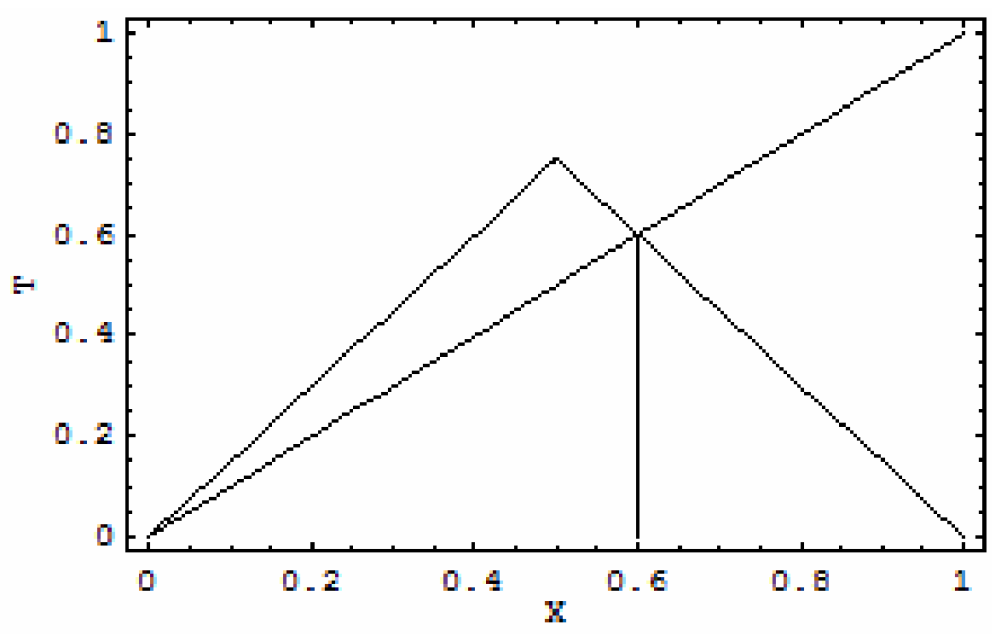

(a)

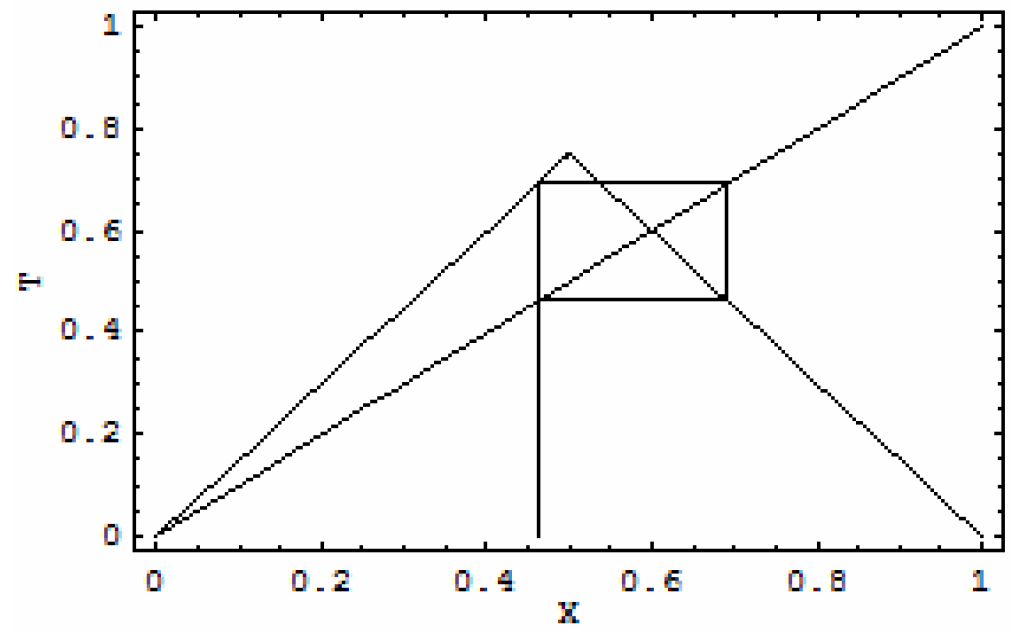

(b) 


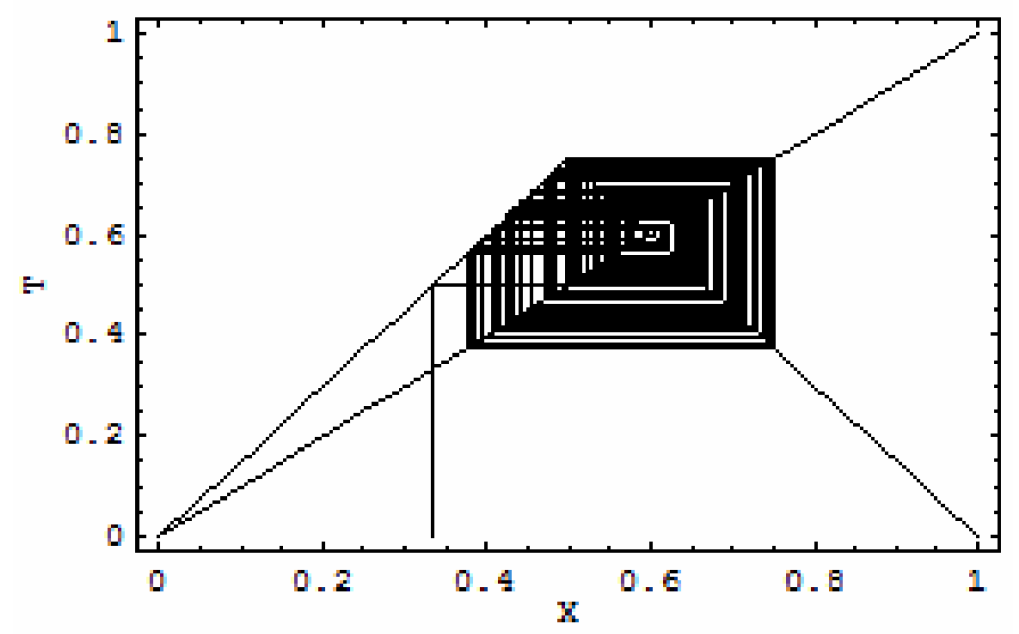

(c)

Figure 3. Graphical iterations when $b=\frac{3}{2}$ : (a) $x_{0}=\frac{3}{5}$, (b) $x_{0}=\frac{6}{13}$, and (c) $x_{0}=\frac{1}{3}$, for 200 iterations.

In case (i), the iterate $x_{n+1}$ is equal to $x_{n}$ for all $n$. This type of sequence displays period-one behaviour. In case (ii) the iterate $x_{n+2}$ is equal to $x_{n}$ for all $n$, and the result is period-two behaviour. In case (iii), the first 11 iterations are listed but other methods need to be used in order to establish the long term behaviour of the sequence.

(IV) $b=2$ :

(i) $\left\{\frac{1}{3}, \frac{2}{3}, \frac{2}{3}, \ldots, \frac{2}{3}, \ldots\right\}$;

(ii) $\left\{\frac{1}{5}, \frac{2}{5}, \frac{4}{5}, \frac{2}{5}, \frac{4}{5}, \ldots, \frac{2}{5}, \frac{4}{5}, \ldots\right\}$;

(iii) $\left\{\frac{1}{7}, \frac{2}{7}, \frac{4}{7}, \frac{6}{7}, \frac{2}{7}, \frac{4}{7}, \frac{6}{7}, \ldots, \frac{2}{7}, \frac{4}{7}, \frac{6}{7}, \ldots\right\}$;

(iv) $\left\{\frac{1}{11}, \frac{2}{11}, \frac{4}{11}, \frac{8}{11}, \frac{6}{11}, \frac{10}{11}, \frac{2}{11}, \ldots, \frac{2}{11}, \frac{4}{11}, \frac{8}{11}, \frac{6}{11}, \frac{10}{11}, \ldots\right\}$. 
The sequences behave as follows: (i) there is period-one behaviour, (ii) there is period-two behaviour, (iii) there is a period-three sequence, and (iv) there is a period-five sequence.

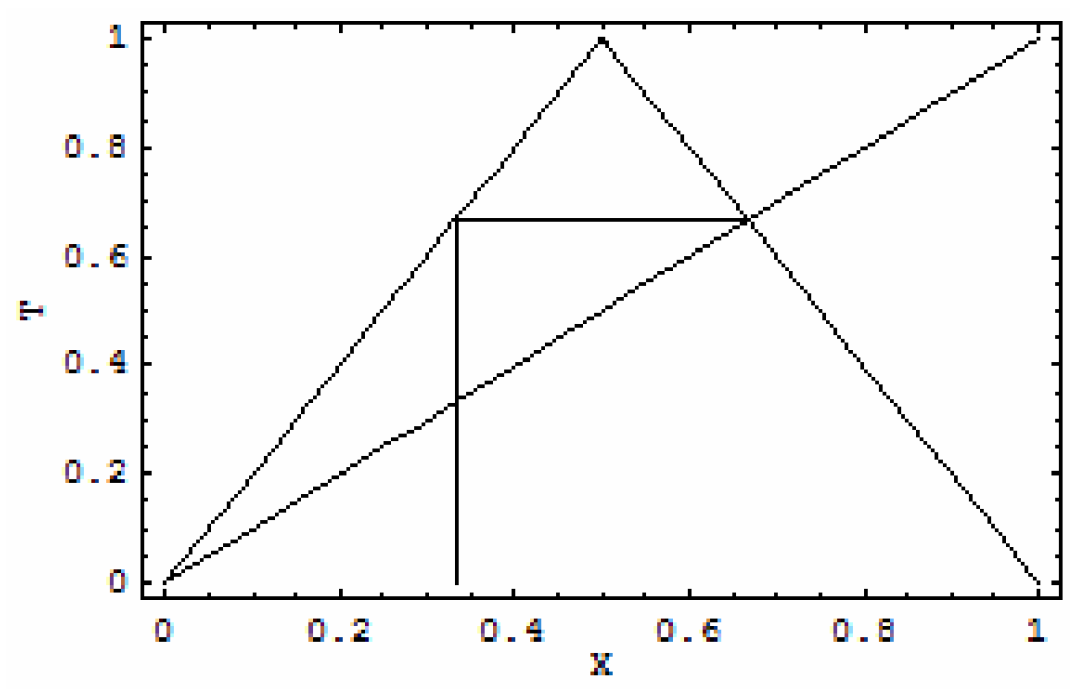

(a)

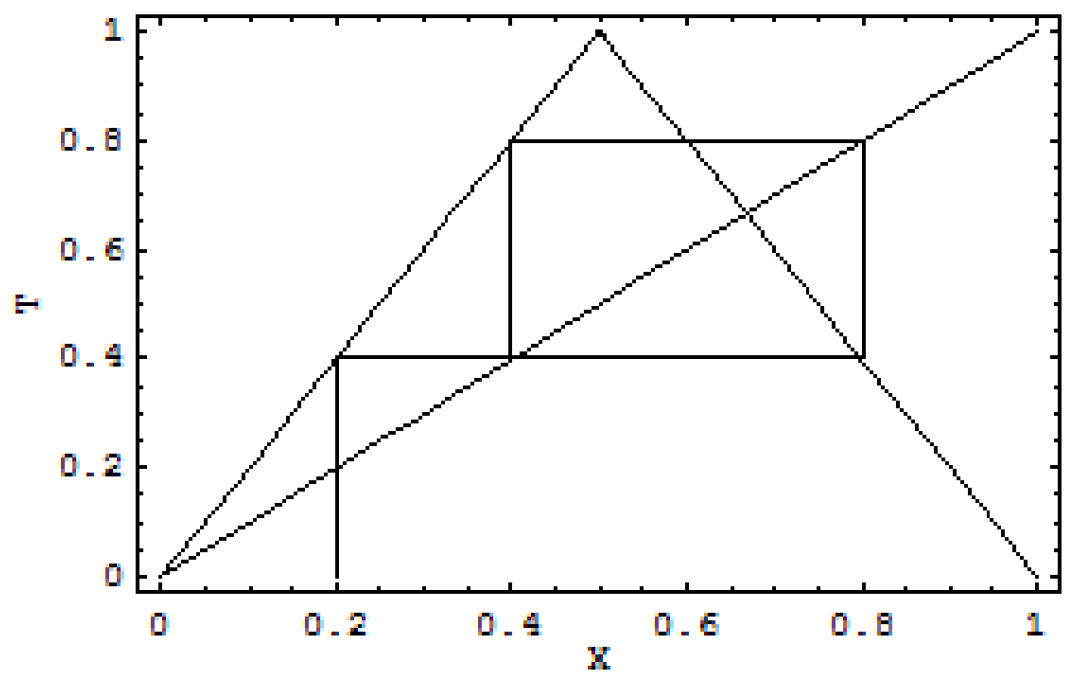

(b) 


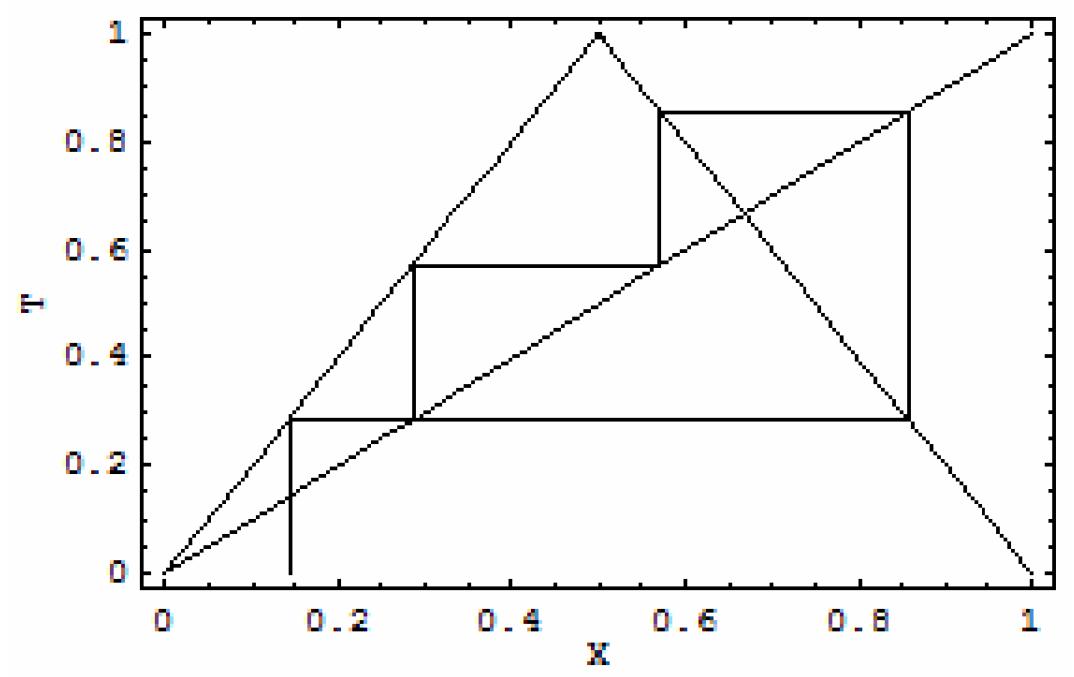

(c)

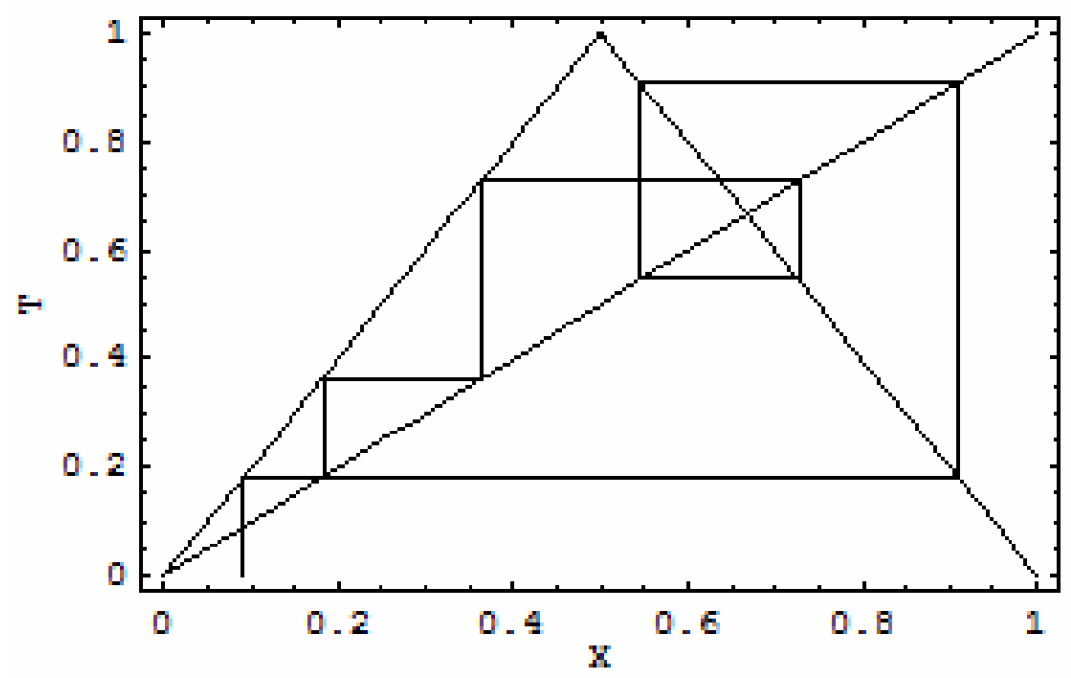

(d)

Figure 4. Graphical iterations when $b=2$ : (a) $x_{0}=\frac{1}{3}$, (b) $x_{0}=\frac{1}{5}$,

(c) $x_{0}=\frac{1}{7}$ and (d) $x_{0}=\frac{1}{11}$, for 200 iterations. 
Again if we iterate the tent function graphically for $b=2:$ (a) $x_{0}=0.2$, and (b) $x_{0}=0.2001$ we can get the following figures.

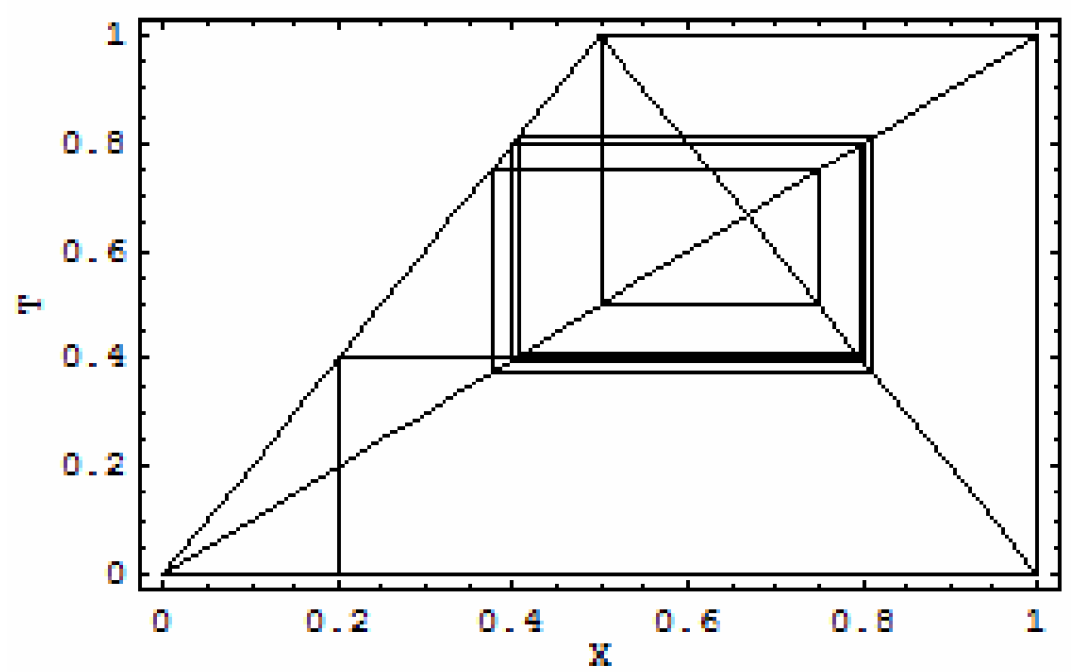

(a)

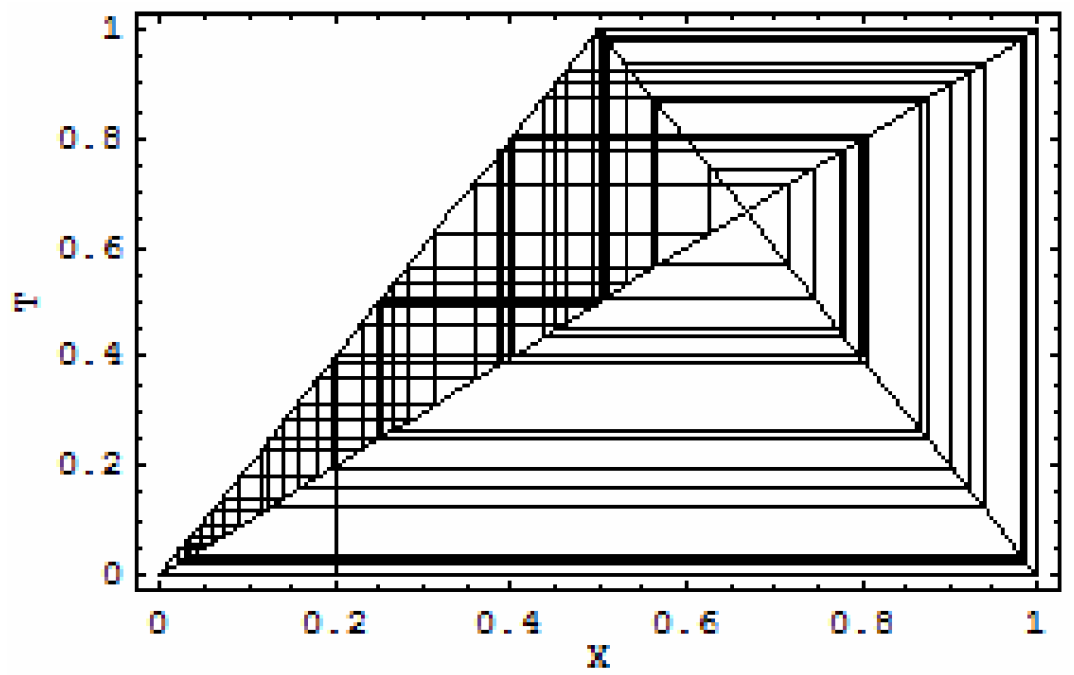

(b)

Figure 5. Graphical iterations when $b=2$ : (a) $x_{0}=0.2$, and (b) $x_{0}=0.2001$, for 200 iterations. 
Each of the diagram (figure) can be reproduced by using

MATHEMATICA. When $b=\frac{3}{2}$, the tent map [11] displays sensitivity to initial conditions and can be described as being chaotic [10]. The iterative path plotted in Figure 3(c) appears to wander randomly. It is still not clear whether the path is chaotic or whether the path is periodic of a very high period. Figure 5 clearly shows the sensitivity to initial conditions. Again it is not clear in case (ii) whether the path is chaotic or of a very high period. It is clear from the diagrams is that the three basic properties of chaos and they are mixing, periodicity, sensitivity to initial conditions and they are all exhibited for certain parameter values. Indeed, a now-famous result due to $\mathrm{Li}$ and Yorke [6] states that if a system displays period-three behaviour, then the system can display periodic behaviour of any period. Li and Yorke then go on to prove that the system can display chaotic phenomena. Hence when $b=2$, system $x_{n+1}=T_{b}\left(x_{n}\right)$ is chaotic since it has a period-three sequence (Problem 1 (iv), (iii)).

\section{Period-doubling of the logistic map}

The logistic map is defined by

$$
f_{a}(x)=a x(1-x), x \in[0,1], a>0 \text { for given } x \in[0,1] .
$$

By varying the parameter $a$, the following behaviour is observed:

(i) $0<a<1$ :

In this case, $f_{a}(x)$ gives us a single fixed point $x=0, x=0$ is attracting (or stable).

(ii) $1<a<3$ :

In this case, solving $f_{a}(x)=x$ gives us two fixed points $x=0$ and $x=1-\frac{1}{a} \cdot f_{a}^{\prime}(0)=a>1 \Rightarrow x=0$ is repelling (or unstable). 


$$
\left|f_{a}^{\prime}\left(1-\frac{1}{a}\right)\right|=|2-a|<1 \Rightarrow x=1-\frac{1}{a} \text { is attracting (or stable) [1]. }
$$

Then we can show that all the pre-images of this interval are $(0,1)$.

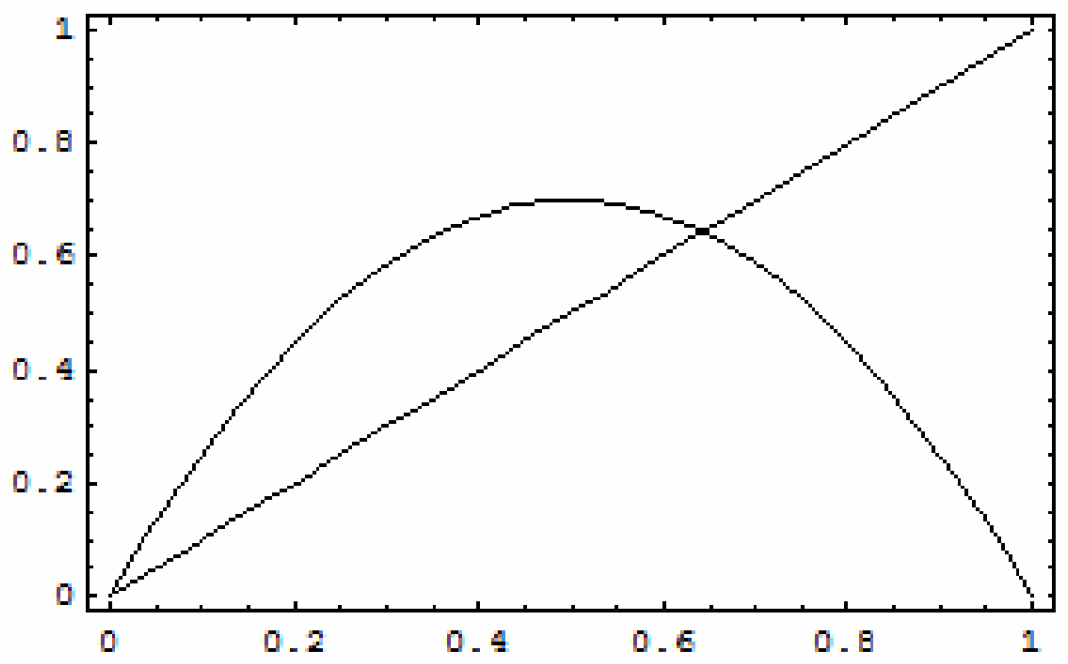

Figure 6. $f_{a}(x)$ for $a=2.8$.

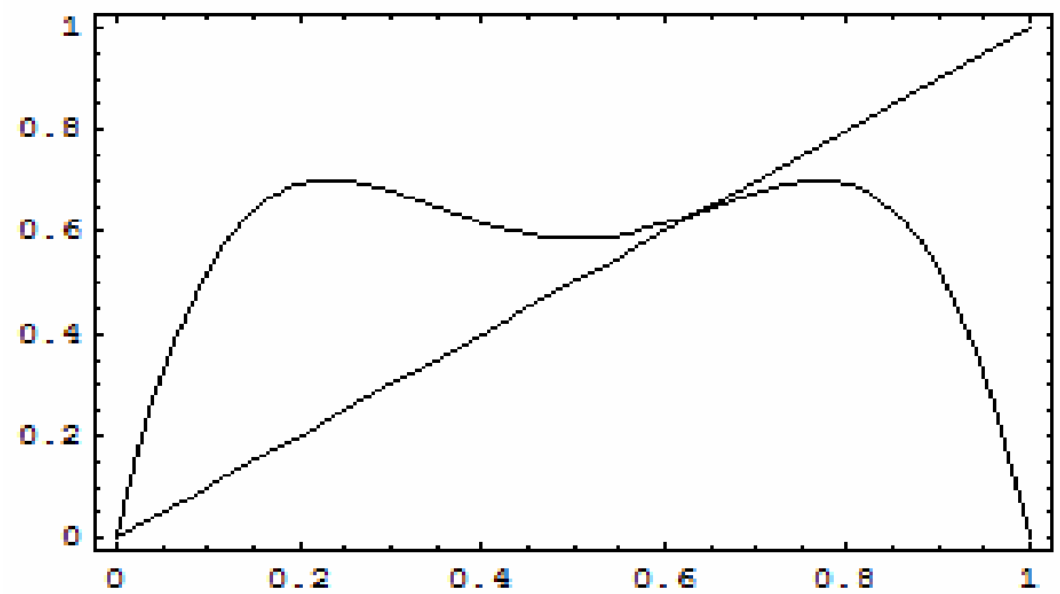

Figure 7. $f_{a}^{2}(x)$ for $a=2.8$. 
(iii) $a>3$ :

In this case, both of the two fixed points are repelling. But the periodic points appear 2-cycle. Solving $f_{a}^{2}(x)=x$ gives us four roots $x=0,1-\frac{1}{a}, \frac{a+1 \pm \sqrt{(a-3)(a+1)}}{2 a}$.

The first two are clearly the repelling fixed points. The second two are two-periodic points. We have $\left(f_{a}^{2}\right)^{\prime}\left(x_{0}\right)=f_{a}^{\prime}\left(x_{1}\right) f_{a}^{\prime}\left(x_{0}\right)$, where $x_{1}=f_{a}\left(x_{0}\right)$.

For $x_{0}=\frac{a+1 \pm \sqrt{(a-3)(a+1)}}{2 a},\left(f_{a}^{2}\right)^{\prime}\left(x_{0}\right)<1$.

Therefore the 2 -cycle is attracting for $\left|4+2 a-a^{2}\right|<1$. That is $3<a$ $<1+\sqrt{ } 6 \approx 3.449 \ldots$.

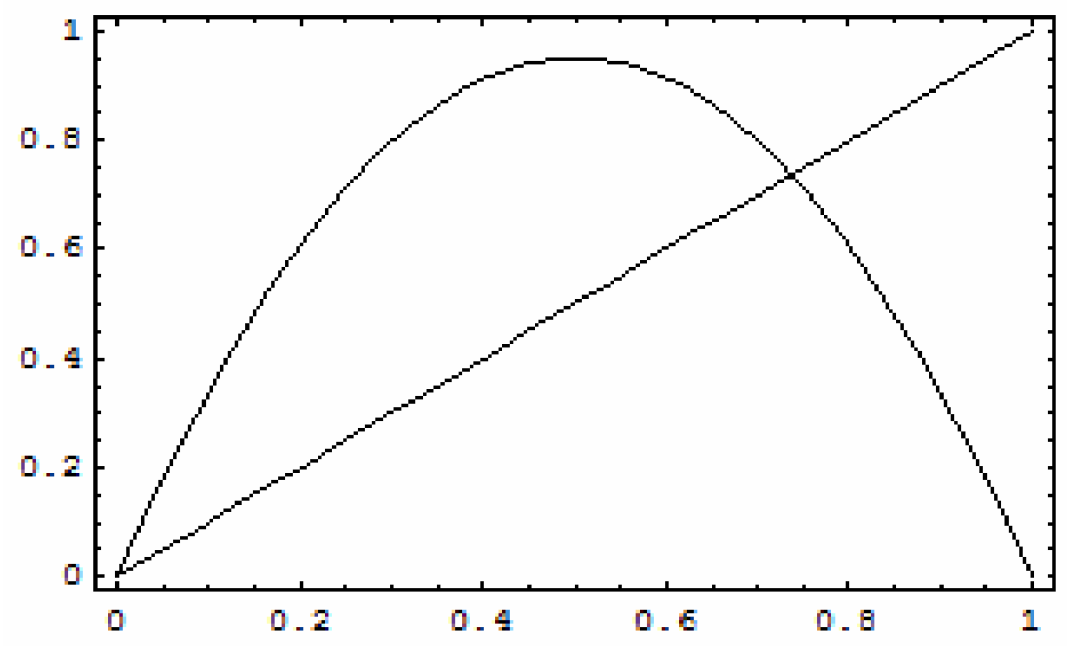

Figure 8. $f_{a}(x)$ for $a=3.8$. 


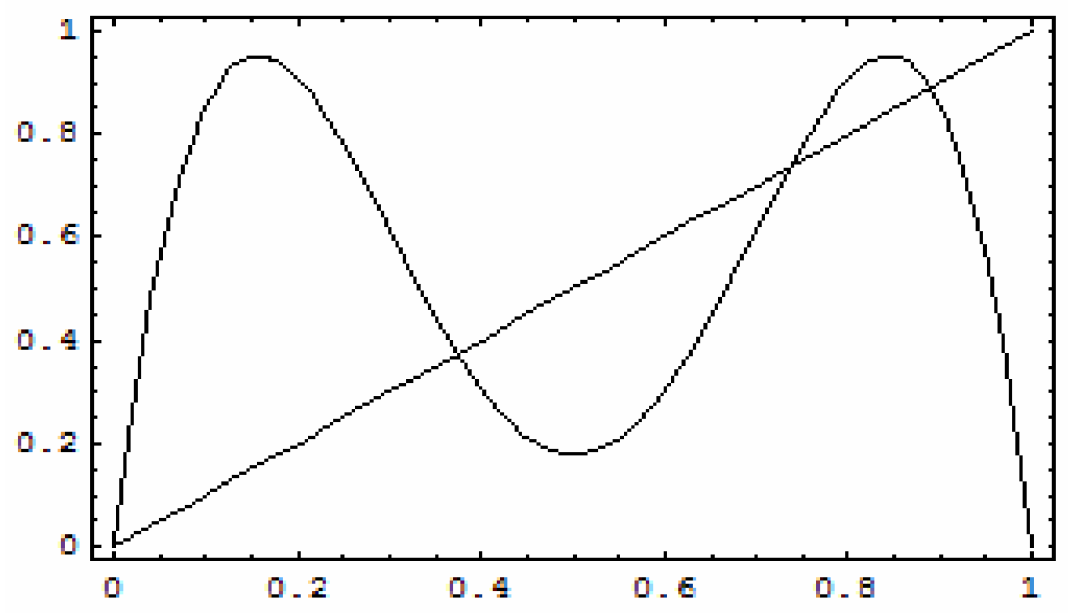

Figure 9. $f_{a}^{2}(x)$ for $a=3.8$.

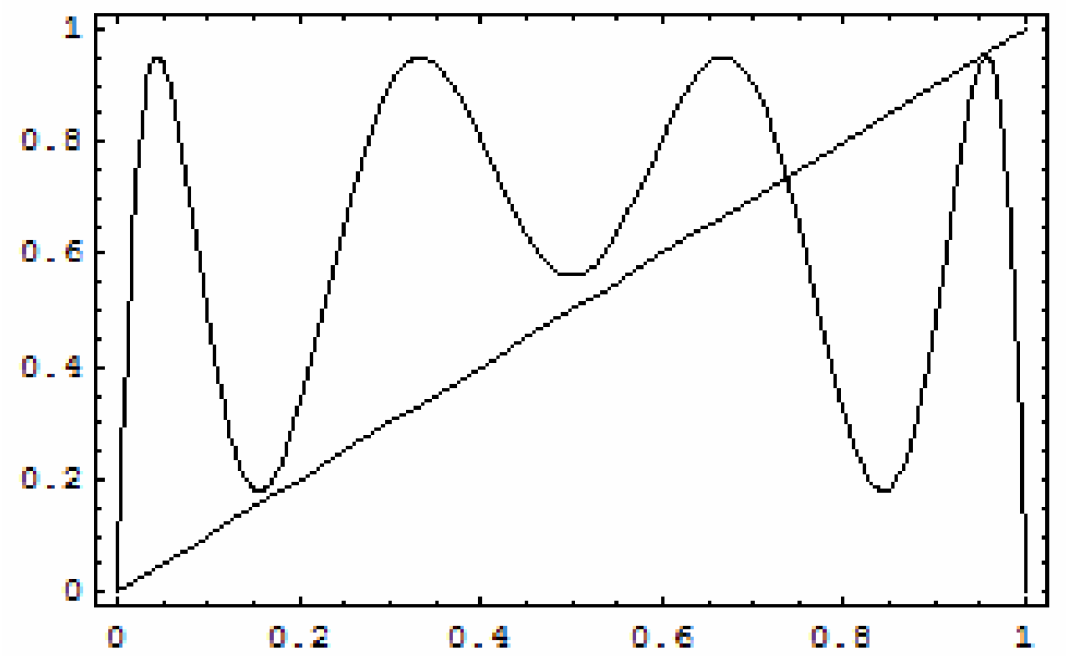

Figure 10. $f_{a}^{3}(x)$ for $a=3.8$. 


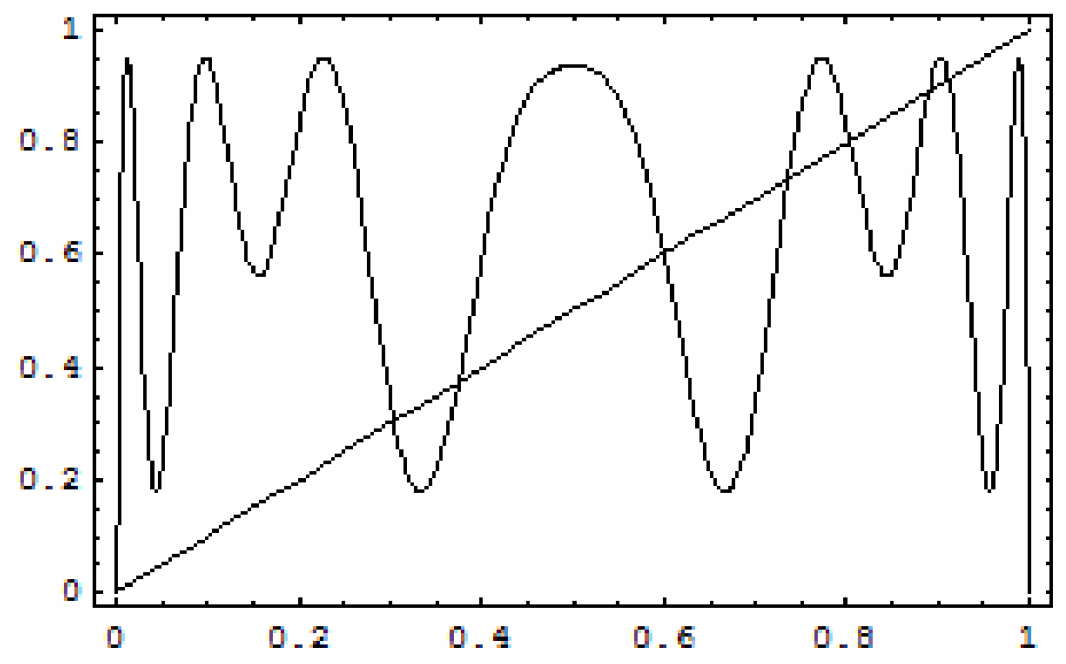

Figure 11. $f_{a}^{4}(x)$ for $a=3.8$.

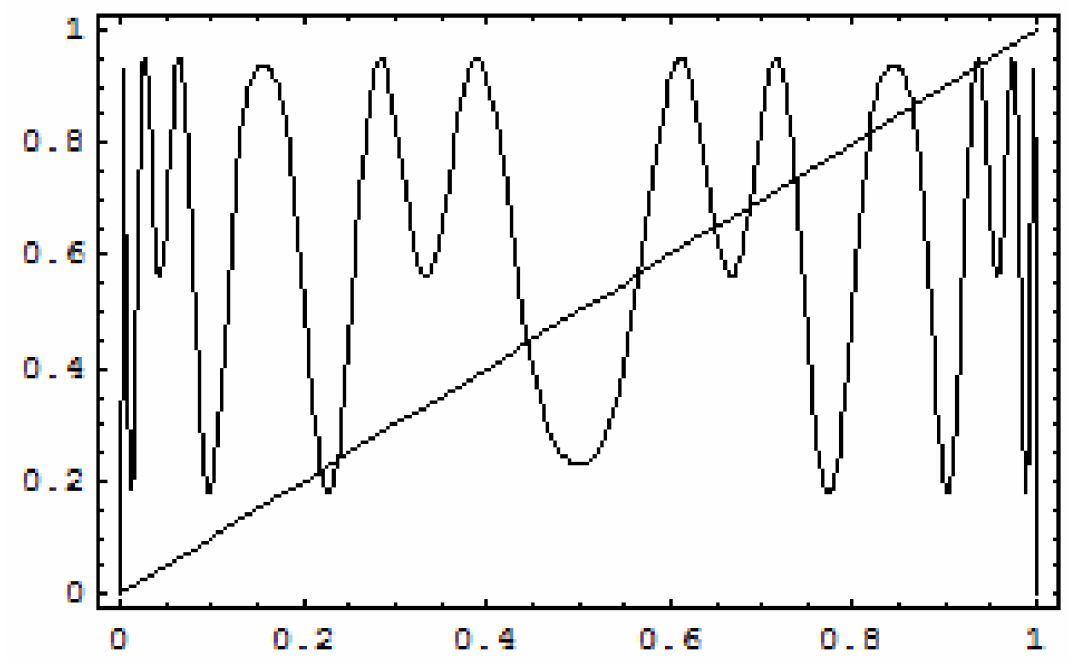

Figure 12. $f_{a}^{5}(x)$ for $a=3.8$. 


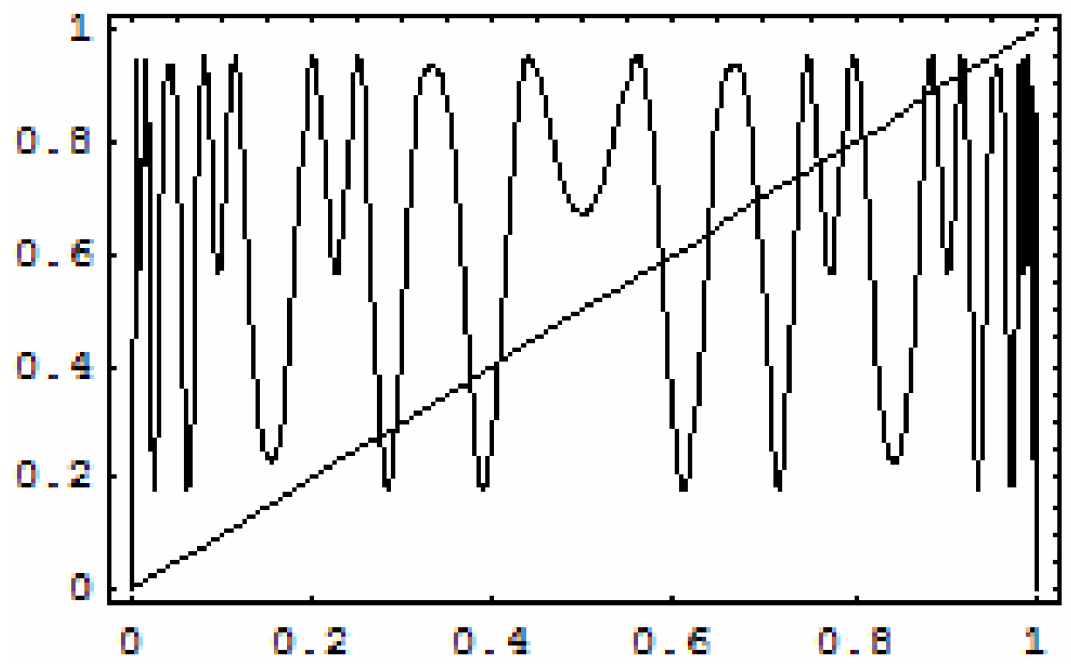

Figure 13. $f_{a}^{6}(x)$ for $a=3.8$.

Every point is eventually attracted to this 2-cycle. Numerically, one usually finds that every point in $[0,1]$ is attracted to this 2 -cycle because both 0 and $1-\frac{1}{a}$ are repelling, when $a>1+\sqrt{6}$ the 2 -cycle is repelling, but a 4-cycle appears $4>a>1+\sqrt{6}=3.449$.

When $3.449 \ldots<a<3.54409 \ldots$ there is an attracting 4-cycle. Numerically every point is eventually attracted to it. When $3.54409 \ldots<a<3.5644 \ldots$, there is an attracting 8-cycle which numerically attracts every point in $(0,1)$. When $3.5644 \ldots<a<3.568759 \ldots$, there is an attracting 16 -cycle. This is the so-called period-doubling bifurcation. Note that the successive bifurcations come faster and faster. Ultimately, the intervals of $2 n$-cycles converge to a point $a_{\infty} \approx 3.569946 \ldots$ as $n \rightarrow \infty$. It is relatively easy to show that the logistic map is chaotic on an invariant Cantor set for $a>2+\sqrt{5} \approx 4.236$, but in fact, it is also chaotic for all $a>4$. 


\section{Bifurcation Diagram of Tent Map}

In this section, we are going to explore the concept of bifurcations and how MATHEMATICA can help us visualize these changes. We will begin with some basic definitions, then examine some plots, and finally create a bifurcation diagram. A bifurcation is a fundamental change in the nature of a solution. When studying dynamical systems, we often want to know when the system undergoes a bifurcation. A bifurcation diagram is a kind of plot that shows the value of the changing parameter, $b$ in our case, on one axis and the solution to the system on the other axis. Our next goal is to produce the bifurcation diagram of the 'tent' maps $T_{b}$. 


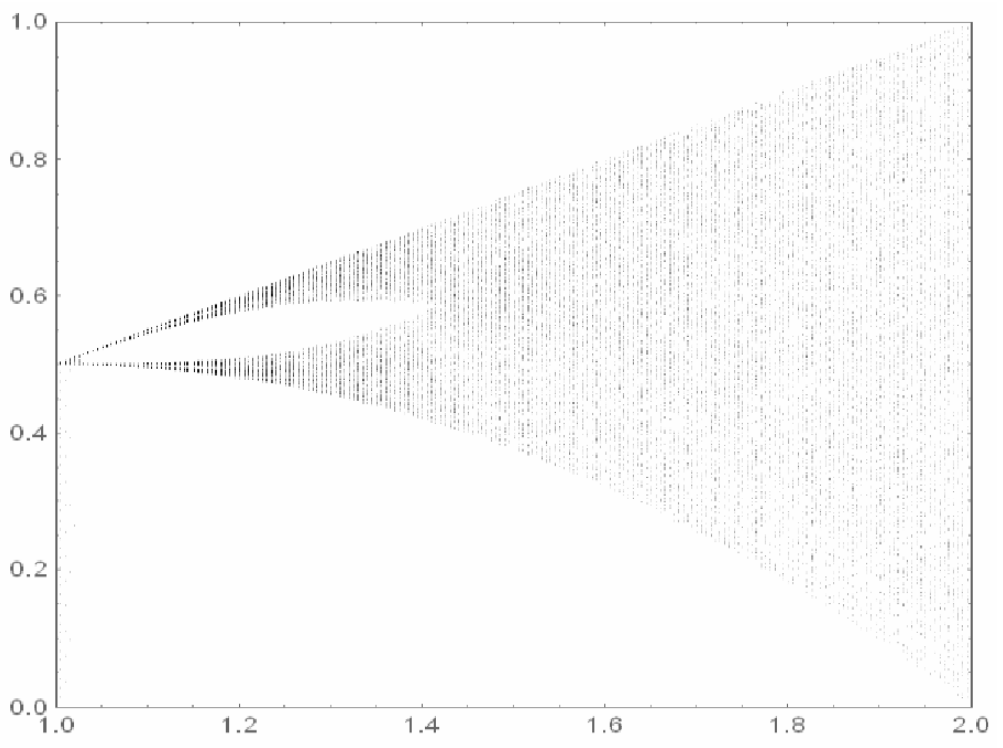

Figure 14(a). Bifurcation diagram of tent map.

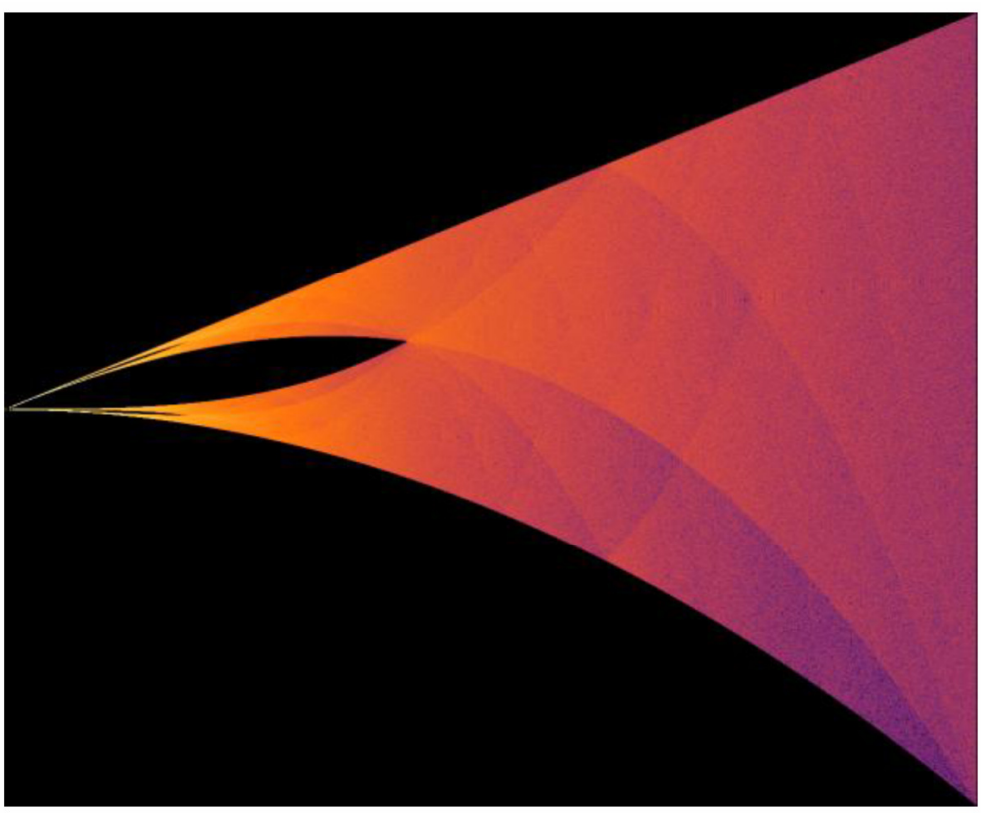

Figure 14(b). Bifurcation diagram of the tent map. 
Often we want to know how system behaviour depends on parameters. In the case of the tent map $T_{b}$, we have a single parameter $b$, and we have already known that there is a stable equilibrium for $b<\frac{1}{2}$. We also know that $b>\frac{1}{2}$, there are two unstable equilibrium, some unstable periodic solutions, and, as we have just seen, chaotic solutions. We can get an overview of how all of this depends on the parameter $r$ by a bifurcation diagram. That is a plot in which the abscissa is the value, and on the ordinate we plot all of the $x$-values from an orbit for that value $b$. Fixed points will show up as a single point, a periodic orbit as several points, and a chaotic orbit as a band or several bands of points [13].

\section{Conclusion}

The aim of this paper is to demonstrate the routes to chaos in dynamical systems and study chaos in dynamical system in terms of some well known maps that exhibits chaotic behaviour and contribute to a few application of chaos in dynamical system. Different kind of chaotic map and their chaotic behaviour with changing values are also studied in this paper. The study of low dimensional map, which exhibit chaotic behaviour is a very active area of current research. In this paper, we see that the equation of the logistic map is not particularly complex, so we might not expect anything to exciting to happen. For values of $a$ less than about 3.45 the value of $x$ will bounce around a few times and quickly converge on a value. However if we keep increasing the value of $a$ things become more interesting. As $a$ continues to increase $x$ will no longer settle to a single value, but instead oscillate between two values. If we keep increasing the value of $a$ we will find that $x$ now starts to oscillate between 4 values, and then 8 then 16 and so on. However, if we keep increasing $a$ we will occasionally see that some values of $a$ again seem to show more ordered behaviour. 
The relative simplicity of the logistic map makes it an excellent point of entry into a consideration of the concept of chaos. In this paper, we observe that a rough description of chaos is that chaotic systems exhibit a great sensitivity to initial conditions -- a property of the logistic map for most values of $a$ between about 3.57 and 4 (as noted above). A common source of such sensitivity to initial conditions is that the map represents a repeated folding and stretching of the space on which it is defined. In the case of the logistic map, the quadratic difference equation describing it may be thought of as a stretching-and-folding operation on the interval $(0,1)$. On the other hand when $b=2$, the tent map has becomes chaotic. Chaos theory is a mathematical theory, and it is still in development. In future multi-dimensional dynamical system and their behaviour can be described more effectively with advanced chaos theory. It will enable the description of a series of phenomena from the field of dynamical system. No one disagree the importance of chaos in dynamical system in our practical and scientific life. Actually now a day chaos in dynamical system is essential in every sector.

\section{References}

[1] H. R. Biswas, One dimensional chaotic dynamical system, Journal of Pure and Applied Mathematics: Advances and Applications 10(1) (2013), 69-101.

[2] F. Blanchard, E. Glasner, S. Kolyada and A. Maass, On Li-Yorke pairs, Journal für die Reine und Angewandte Mathematik 547 (2002), 51-68.

DOI: https://doi.org/10.1515/crll.2002.053

[3] R. L. Devaney, A First Course in Chaotic Dynamical Systems, Perseus Press, 1993.

[4] R. L. Devaney, An Introduction to Chaotic Dynamical Systems, 2nd Edition, Boulder, Co. Westview Press, 2003.

[5] Edward Ott, Chaos in Dynamical Systems, Cambridge University Press, 2002.

[6] T. Y. Li and J. A. Yorke, Period three implies chaos, American Mathematical Monthly 82(10) (1975), 985-992.

DOI: https://doi.org/10.1080/00029890.1975.11994008

[7] R. A. Holmgren, A First Course in Discrete Dynamical Systems, Springer-Verlag, 1996.

[8] Robert C. Hilborn, Chaos and Nonlinear Dynamics, Oxford University Press, 2000. 
[9] Samuel Effah-Poku, Chaos in Dynamical System, Kwame Nkrumah University of Science and Technology, Kumasi, M.phil Thesis, 2017.

[10] S. H. Strogatz, Nonlinear Dynamics and Chaos, Addison-Wesley, Reading MA, 1994, p. 353 .

[11] S. H. Strogatz, Nonlinear Dynamics and Chaos, Perseus Books, 1994.

[12] Shihai Li, $\omega$-Chaos and topological entropy, Transactions of the American Mathematical Society 339(1) (1993), 243-249.

DOI: http://dx.doi.org/10.2307/2154217

[13] Wadia Faid Hassan Al-Shameri and Mohammed Abdulkawi Mahiub, Some dynamical properties of the family of tent maps, International Journal of Mathematical Analysis 7(29) (2013), 1433-1449.

DOI: http://dx.doi.org/10.12988/ijma.2013.3361 